\section{Resumen}

En este documento analizamos las actividades de integración desarrolladas por futuros profesores de biología, en quinto y sexto semestre del Proyecto Curricular de Licenciatura en Biología (PCLB) de la Universidad Pedagógica Nacional (Colombia), en particular, la construcción de Conocimiento Profesional del Profesor de Biología. Dichas actividades constituyen el diseño de unidades didácticas (UD) para la enseñanza de una temática de biología y la formulación de un Anteproyecto de la Práctica Pedagógica (APP) sobre educación en biología.

Para ello, el estudio se concentra en dos categorías: Conocimiento Biológico (CB) y Conocimiento Profesional del Profesor de Biología (CPPB). Asimismo, el diseño de unidades didácticas posibilitó en los futuros profesores identificar e integrar los diferentes conocimientos y saberes que requiere el docente para enseñar, así como problematizar la enseñanza a partir del Conocimiento Biológico, la Pedagogía y la Didáctica, y la Epistemología de la Biología; lo cual conduce a la estructuración de contenidos, propósitos, evaluación y actividades de enseñanza.

Esta estrategia favoreció principalmente la construcción de Conocimiento Didáctico del Contenido Biológico (CDCB). Para el caso de la formulación del APP, los futuros profesores reconocieron y reflexionaron sobre el saber construido en relación con la profesión docente durante los primeros seis semestres del PCLB, en el cual se destaca la fuente académica y experiencial para la construcción del conocimiento profesional.

\section{Palabras clave}

Conocimiento profesional del profesor de biología, conocimiento didáctico del contenido biológico, formación docente, conocimiento biológico, futuros profesores de biología, anteproyecto de la práctica pedagógica.

\section{Abstract}

This paper analyzes integrative learning activities developed by future Biology teachers of fifth and sixth semester enrolled in the Biology teacher training program (PCLB) at Universidad Pedagógica Nacional (Colombia), who are building up a professional knowledge for the Biology teacher. These activities are didactic units (UD) designed for teaching any topic of Biology and a statement of the First Draft of Pedagogical Practice (APP) on Biology training. The analysis focuses on two categories: Biology Knowledge (CB) and Professional Knowledge of the Biology Teacher (СРPB). The analysis shows that the design of didactic units allows future teachers to identify and integrate several types of knowledge and learning aspects required by the teaching situation. In addition, it allows them to identify problems and solve them regarding education from the point of view of Biology knowledge, the Pedagogy and Didactics, and the Epistemology of Biology, which leads to structure teaching contents, goals, assessment, and activities. This strategy has mainly favored to build up a didactic knowledge of biology contents (CDCB). Regarding an APP statement, future teachers recognize and reflect themselves on knowledge built in relation to the teaching profession during first six semesters of the program.

\section{Key words}

Biology teacher's professional knowledge, didactic knowledge of biology contents, teaching training, biology knowledge, future biology teachers, first draft of pedagogical practice. 


\title{
Procesos formativos que favorecen la construcción del conocimiento profesional del profesor en futuros docentes de Biología ${ }^{1}$
}

\author{
Édgar Orlay Valbuena Ussa² \\ Alice Marcela Gutiérrez Pérez ${ }^{3}$ \\ Mónica Alexandra Correa Sánchez ${ }^{4}$ \\ Elías Francisco Amórtegui Cedeño ${ }^{5}$
}

Un problema relevante, en el campo de la didáctica de las ciencias, es la formación de profesores, tanto, que se ha consolidado como línea de investigación (Astolfi, 1994; Porlán, 1998; Gil, Carrascosa y Martínez, 1999, 2000; Porlán, Rivero y Martín del Pozo, 2000; Gallego, 2004). Al respecto, una de las preocupaciones sustanciales es cómo modificar la representación mayoritaria reduccionista, consistente en que para enseñar una asignatura basta con que el profesor posea un dominio de la disciplina científica específica, por ejemplo, la idea según la cual es suficiente tener dominio de la materia para ser excelente profesor de ésta.

\footnotetext{
${ }^{1}$ Este artículo recoge los resultados de la investigación "Construcción del conocimiento profesional", del profesor de biología en el Proyecto Curricular de Licenciatura en Biología de la Universidad Pedagógica Nacional, desarrollada durante 2007 y 2008 con financiación del Centro de Investigaciones de la Universidad Pedagógica Nacional, CIUP.

Texto recibido el 18 de junio, evaluado el 23 y el 24 de junio y arbitrado el 30 de junio de 2009.

2 Doctor en Didáctica de las Ciencias Experimentales, Universidad Complutense de Madrid. Profesor de planta, Departamento de Biología, Universidad Pedagógica Nacional de Colombia. Coordinador Línea de Investigación Conocimiento Profesional del Profesor de Ciencias. edgarorlay@hotmail.com valbuena@pedagogica.edu.co

${ }^{3}$ Magíster en educación, Pontificia Universidad Javeriana. Profesora ocasional, Departamento de Biología, Universidad Pedagógica Nacional. galice_marcela@hotmail.com

${ }^{4}$ Estudiante décimo semestre del Proyecto Curricular de Licenciatura en Biología, Universidad Pedagógica Nacional. Docente Instituto Pedagógico Arturo Ramírez Montúfar, IPARM. macs1986@hotmail.com

${ }^{5}$ Estudiante décimo semestre del Proyecto Curricular de Licenciatura en Biología, Universidad Pedagógica Nacional.folkerpan@hotmail.com mfed_eamortegui127@pedagogica.edu.co
} 
Desde esta perspectiva, se han realizado variadas investigaciones con profesores de ciencias, tanto en ejercicio como en formación inicial (especialmente con los primeros, véase Porlán y Rivero, 1998; Gess-Newsome y Lederman, 1999). Dichos estudios se han ocupado principalmente del análisis de las características de los componentes y fuentes del conocimiento que diferencian al profesor de ciencias, lo cual corresponde con las producciones de la línea de investigación de Conocimiento Profesional del Profesor, y se ha desarrollado a partir de la década del ochenta del siglo XX con aportes de investigadores como Shulman, Grossman, Carlsen y Marcelo, entre otros (Shulman, 1986a, 1986b; Grossman, 1990; Carlsen, 1999; Marcelo, 1999).

No obstante, para el caso particular de los docentes de biología, son escasas las investigaciones sobre el CPPB. Los aportes son hasta el momento, apenas incipientes; los estudios enfatizan en el Conocimiento Biológico que poseen los profesores y su relación con la enseñanza (Hashweh, 1987; Hoz, Tomer, y Tamir, 1990; Hauslein, Good y Cummins, 1992; Gess-Newsome y Lederman, 1993; Lederman, GessNewsome y Latz, 1994; Gess-Newsome y Lederman, 1995) y muy pocos se centran en los saberes y conocimientos particulares que requiere el docente para enseñar esta ciencia, es decir, en el Conocimiento Didáctico del Contenido Biológico; de hecho, en la revisión realizada sólo aparecen dos investigaciones en las que se hace explícito el estudio del CDCB (Veal y Kubasko, 2003; Valbuena 2007).

Aunque la revisión realizada sobre el CPPB reporta investigaciones que tratan fundamentalmente la caracterización de aquellos saberes y conocimientos que poseen los docentes de biología y que los faculta para enseñar, lo cual aporta elementos importantes para tener en cuenta en la formación inicial y permanente de profesores, en particular en cuanto a los contenidos formativos que implica.

De igual manera, se plantea que es relevante investigar la incidencia de los procesos formativos en la construcción de dicho conocimiento. En ese sentido, nos circunscribimos en un programa curricular de formación inicial de profesores de biología, concretamente en el Proyecto Curricular de Licenciatura en Biología de la Universidad Pedagógica Nacional, dado que los autores de este artículo estamos involucrados en él (dos en calidad de formadores y dos en calidad de docentes en formación).

Con base en estos elementos, se plantea la siguiente pregunta de investigación: ¿Cómo se contribuye a la construcción de Conocimiento Profesional del Profesor de Biología en el Proyecto Curricular de Licenciatura en Biología? Desde esta perspectiva, la investigación se ha focalizado en caracterizar y analizar los factores que pueden facilitar u obstaculizar la construcción de dichos saberes y conocimientos propios del CPPB. Aunque los docentes en formación no tengan experiencia como profesores, es innegable que la mayor parte de su vida han permanecido en las aulas. Ello supone que a partir de dicha experiencia construyen referentes epistemológicos acerca de la enseñanza, el aprendizaje, las metodologías y actividades de enseñanza, la evaluación de los aprendizajes, etc. 
De este modo, al relacionar la formación inicial docente con la perspectiva de construcción de conocimiento profesional del profesor (CPP), es importante resaltar la incidencia de los procesos formativos en el cambio de referentes de los profesores acerca de dicho conocimiento. Así, en el ámbito de formación docente vale la pena investigar aquellos procesos formativos que promuevan la construcción de CPP. Por consiguiente, investigar la construcción de $\mathrm{CPPB}$, en la formación inicial, es condición sine qua non para conocer y analizar lo que futuros docentes puedan experimentar con respecto a su propio proceso educativo, junto con sus motivaciones, proyecciones y reflexiones.

En primera instancia, existe una identificación con Tardif (2009), quien define el saber del profesor a partir del postulado de la subjetividad, en el cual establece que los profesores en el contexto de sus tareas cotidianas poseen, utilizan y producen saberes específicos de su profesión; es decir, los maestros son actores competentes y sujetos de conocimiento, no simples ejecutores técnicos de los conocimientos producidos por otros (Valbuena, 2007). En efecto, los significados y desarrollos que llevan a cabo los profesores no son solamente producto de lo que han estudiado, también forman parte de interpretaciones, reflexiones y elaboraciones que -en y sobre la práctica- han tenido que hacer, lo cual fundamenta su desarrollo profesional.

Antes de la exposición de aspectos metodológicos, resultados y conclusiones de la investigación, se presentarán los principales elementos teóricos en los que se enmarca el estudio, los cuales hacen referencia al Conocimiento Profesional del Profesor y al Conocimiento Biológico.

\section{Conocimiento profesional del profesor}

Debido a la complejidad tanto conceptual como contextual de la enseñanza, para el caso del Conocimiento Profesional del Profesor (CPP) resulta complicado identificar los componentes que lo integran y vislumbrar su estructura. Las investigaciones al respecto diferencian cuatro grandes componentes: conocimiento disciplinar, conocimiento pedagógico, conocimiento didáctico del contenido (CDC) y conocimiento del contexto.

En primer lugar, el conocimiento disciplinar contempla los contenidos concretos que se enseñan de una disciplina en particular, así como la manera en que están organizados en estructuras sustantivas y sintácticas, según la clasificación de Joseph Schwab (1978, citado en Shulman, 1986a, 1986b). El conocimiento pedagógico quizás es el más conocido, teniendo en cuenta los numerosos estudios realizados acerca de varios de sus aspectos (Grossman, 1990), y asimismo es común para la enseñanza de diferentes saberes.

Algunos modelos del conocimiento profesional docente como el de Morine, Dershimer y Kent (1999) hacen distinción entre una pedagogía general y otra personal, esta última marcada por el saber experiencial. El conocimiento del contexto influye en el resto de componentes, pero especialmente en el CDC, ya que el cono- 
cimiento cotidiano está determinado por la relación del sujeto con los elementos contextuales en diferentes niveles (sociedad, cultura, familia, escuela, etc.). Por su parte, el conocimiento didáctico del contenido trata el qué, el cómo y el para qué enseñar determinada disciplina. Diferentes investigadores coinciden en que este dominio es el que realmente identifica al saber profesional, pues genera un mayor impacto en las acciones de enseñanza en el aula de clase (Grossman, 1990; Carlsen, 1999; Morine-Dershimer y Todd Kent (1999); Valbuena, 2007).

Asimismo, se han propuesto diferentes modelos de CPP (Shulman, 1986a, 1986b; Bromme, 1988; Grossman, 1990; Porlán et al., 1996; Magnusson, Krajcik y Borko, 1999; Morine-Dershimer y Todd Kent, 1999; Carlsen, 1999, entre otros). Aquí se hace referencia al modelo del conocimiento profesional como teoría y práctica, con el cual hay identificación. Dicho modelo ha sido propuesto por el grupo español Didáctica e Investigación Escolar (DIE), gestor del proyecto curricular Investigación y Renovación en la Escuela (IRES) (Porlán et al, 2001).

En efecto, el CPP se entiende como la interrelación e integración sistémica y compleja (que requiere un profundo proceso de reelaboración y transformación epistemológica y didáctica) de saberes de distinta índole, el cual se genera a partir de situaciones y problemas prácticos del contexto educativo particular. Dicho conocimiento se asume como una transformación formativa desde una perspectiva evolutiva, en la que se formula una hipótesis de progresión con miras a reestructurar, de forma continua y compleja el conocimiento de hecho del profesor en un conocimiento deseable.

Así, los productos de esa reelaboración y transformación se constituyen como sistemas de ideas en evolución, de carácter tentativo, procesual y evolutivo (Porlán et al, 1996; García, 1998; Porlán y Rivero, 1998). Este estudio se identifica con este modelo, dado que se fundamenta en las perspectivas compleja, crítica y constructivista, las cuales corresponden con el enfoque epistemológico y ontológico del Proyecto Curricular de Licenciatura en Biología de la Universidad Pedagógica Nacional (Departamento de Biología, 1999). Al respecto, se debe aclarar que la formación inicial de profesores de biología se entiende como un proceso investigativo en el que se busca que el futuro profesor formule, reestructure y resuelva problemas de la educación en esta asignatura, de manera que trasciendan la simple lógica disciplinar científica.

\section{El conocimiento biológico}

En cuanto a la construcción del Conocimiento Profesional del Profesor de Biología, una fuente fundamental está constituida por el conocimiento y las concepciones que posea el profesor acerca del conocimiento disciplinar, es decir, acerca de la biología. Como se señaló previamente, existe una identificación con el modelo de CPP del grupo DIE, el cual sustenta que una perspectiva para el estudio del conocimiento y las concepciones es la utilización de la hipótesis de progresión. Ésta consiste en 
identificar y formular diversos niveles tentativos de progresiva complejidad, desde un nivel inicial correspondiente al "conocimiento de hecho" del profesor (con el que posee) hasta un nivel de referencia, es decir, un "conocimiento deseable".

Dada la extensión que demanda abordar las características del Conocimiento Biológico, se presenta a continuación la elaboración desarrollada por el grupo en investigaciones anteriores (Valbuena, Castro y Sierra, 2006; Valbuena et al, 2006; Sánchez, 2007 y Valbuena, 2007). En este sentido, se propone una hipótesis de progresión para el CB, el cual -de acuerdo con lo reportado por Valbuena, Castro y Sierra (2006)- se puede analizar desde tres perspectivas: características del Conocimiento Biológico, Producción del Conocimiento Biológico y el Objeto de la Biología.

Respecto a las características del Conocimiento Biológico, el nivel inicial corresponde a una realidad objetiva preexistente e independiente del sujeto, con un contenido y un sentido propios. Éste se refiere a generalizaciones de composición, estructura, funcionamiento y clasificación de la vida, principalmente desde la perspectiva fisicista. Las generalizaciones se reflejan en leyes y teorías expresadas en formulaciones matemáticas. En relación con el estatus epistemológico de la biología, no hay una identidad propia al considerarse como una "ciencia auxiliar" que dependa totalmente de ciencias como la física y la química.

En el nivel de referencia, la realidad es un producto dinámico de la interacción que un individuo establece con su entorno social y natural. A la biología se le asigna un estatus epistemológico diferenciado; dadas las especificidades (prácticamente irrepetibles) y cambios (tanto individuales como generacionales) que ocurren en los fenómenos vivientes, no siempre es posible hacer generalizaciones de leyes, teorías y expresiones matemáticas. El Conocimiento Biológico es interdisciplinario y aplicable; no es exacto, sino relativo, diverso, multicausal, individual y particular.

En lo que atañe a la producción del Conocimiento Biológico, el nivel inicial de la hipótesis de progresión corresponde con una visión empírico-positivista, al considerar que este conocimiento se produce a partir de la observación o experimentación de los seres vivos o de los fenómenos biológicos, o mediante la implementación del método científico. La fuente de conocimiento está en lo observado, es decir, en la vida, y trata entonces de hacer una transferencia directa del conocimiento desde el objeto mediante la percepción. Éste corresponde con una visión analítica en la que prima la observación (o experimentación) de las partes, independientemente de la manera en que éstas interactúen.

En el nivel de referencia se tiene en cuenta que, dadas las particularidades de lo vivo, no se pueden unificar metodologías para la producción del Conocimiento Biológico. Al existir especificidades y diferencias entre las unidades de lo vivo en sus diversos niveles, resulta difícil garantizar uniformidad en las réplicas de determinado experimento, con lo cual las condiciones experimentales presentan inconvenientes. Dadas las características de los seres vivos, no es posible usar la categoría explicativa de la causalidad simple. Así, existen diferentes metodologías para la producción del 
Conocimiento Biológico; en el caso de los fenómenos de causación próxima (p. ej. en fisiología, citología, embriología, etc.) se acoge prioritariamente la experimentación con las limitaciones inherentes.

En los estudios que involucran causaciones remotas (por ejemplo, la evolución) se acude principalmente a los métodos comparativos, a la narración histórica. Además, a diferencia de los enfoques fisicistas, en biología los conceptos son fundamentales para la formulación de teorías. En este caso, prima el estudio de las relaciones entre los elementos constituyentes de los sistemas vivientes.

\section{Metodología}

Con el fin de caracterizar y analizar los elementos formativos que contribuyen a la construcción del CPPB, en el Proyecto Curricular de Licenciatura en Biología de la Universidad Pedagógica Nacional, y conscientes de la dificultad de abarcar todos los espacios y actividades formativas, decidimos profundizar en las actividades de integración que se realizan en quinto y sexto semestre, los cuales consisten en el diseño de unidades didácticas (UD) para la enseñanza de temáticas biológicas y la formulación del Anteproyecto de la Práctica Pedagógica (APP), respectivamente. Para efectos de la investigación, la selección presentó lo siguiente:

- El diseño de dos UD (Grupo 1: propuesta para la enseñanza del concepto crecimiento y Grupo 2: propuesta para la enseñanza del concepto fotoirritabilidad). Cada UD fue diseñada por grupo de tres estudiantes.

- La formulación de cuatro APP, cuyas propuestas se titulan: Contribuciones de la Propuesta Pedagógica de la EEPE al aprendizaje significativo del concepto ciclo de vida (estudiante 1-E1); Aspectos teóricos, metodológicos y didácticos que se deben considerar al momento de formular una propuesta de educación ambiental orientada al desarrollo sostenible de una comunidad (estudiante 2-E2); El contacto multisensorial con la naturaleza como estrategia de observación (estudiante 4 -E4); Actitudes que se pueden generar en los estudiantes con respecto a la conservación de especies tomando como referente las ranas en la Sabana de Bogotá $y$ sus alrededores (estudiante 5-E5). Cada APP fue formulado por un estudiante.

Desde el punto de vista metodológico, esta investigación se identifica con la perspectiva de investigación cualitativa con base en un enfoque interpretativo. Los datos analizados provinieron fundamentalmente de los documentos producidos por los futuros docentes durante los procesos de diseño de unidades didácticas y formulación del APP, es decir, dichos informes constituyeron la fuente de información de la investigación. La sistematización de los datos se realizó teniendo en cuenta el 
método de análisis de contenido (Bardín, 1977), que consiste en el siguiente proceso, el cual fue implementado en la investigación de Valbuena (2007):

- Extracción de las unidades de información con sentido, a partir de cada una de las fuentes.

- Agrupación de las unidades de información en las diferentes categorías de investigación (Conocimiento Profesional del Profesor de Biología, Conocimiento Biológico y Aspectos Curriculares relacionados con la construcción del Conocimiento Profesional del Profesor de Biología) con sus respectivas subcategorías.

- Formulación de proposiciones para cada grupo de unidades de información. Dichas proposiciones constituyen una síntesis con bajo nivel de inferencia.

- Análisis de las proposiciones.

\section{Resultados}

Dadas las limitaciones en la extensión de este artículo, y a pesar de la riqueza de información obtenida y sistematizada en la investigación, aquí sólo se destacan los resultados más generales para las dos experiencias de formación, concretamente en lo que atañe a las categorías CPPB y CB. En cada caso se contrastan los resultados con referentes teóricos; luego una reflexión referente a la incidencia de los procesos formativos en la construcción del CPPB, así como las implicaciones de los hallazgos y las sugerencias para aportar al mejoramiento en la formación inicial de profesores de biología. Antes de presentar los resultados de las categorías de investigación, una descripción sucinta de las actividades formativas objeto del estudio.

\section{Descripción de las actividades de integración}

Es menester precisar que cada grupo de trabajo, durante el proceso de diseño de las unidades didácticas, durante las 16 semanas del semestre, elaboró cuatro documentos a medida que avanzaban en el proceso. De igual manera, se aclara que en el transcurso de la formulación del APP cada docente en formación elaboró tres informes, los cuales fueron retroalimentados por los formadores de los diferentes componentes vinculados al semestre.

Durante el proceso, los futuros profesores explicitaron en los diferentes informes: tema de enseñanza, título de la unidad didáctica, objetivos formativos, es decir, lo que le aporta el diseño de la UD en la formación como profesores de biología, justificación en cuanto a la relevancia del tema de enseñanza, importancia para la formación docente, referentes normativos, importancia de la enseñanza del tema, antecedentes, trabajos relacionados con la enseñanza de la temática, esto es, revisión de libros de texto, revistas y libros de enseñanza de las ciencias en los que se publi- 
quen investigaciones referentes a la enseñanza de la temática, además de la caracterización del grupo de alumnos a los que se dirige el material.

Por otra parte, se tuvo en cuenta la elaboración y la sustentación de una red de conceptos biológicos involucrados en la temática escogida, junto con la elaboración y la sustentación pedagógica de una red de contenidos de enseñanza que argumentan las transformaciones didácticas de los diferentes conocimientos y la organización de los diversos contenidos de enseñanza: conceptos, actitudes y procedimientos, formulación de objetivos de enseñanza, así como organización y secuenciación de contenidos y actividades de enseñanza.

Además, los docentes en formación elaboraron el material didáctico como tal, es decir, la unidad didáctica en formato dirigido a los estudiantes de educación básica. Con base en esta actividad de integración, los formadores, además de desarrollar las habilidades procedimentales particulares del diseño de UD, pretenden generar un espacio reflexivo en el que los docentes en formación se piensen como profesores, identificando lo que debe saber y hacer un maestro para enseñar con nivel profesional.

Por su parte, en sexto semestre, los futuros docentes formulan anteproyectos de la Práctica Pedagógica que dan cuenta de sus desarrollos conceptuales, procedimentales y actitudinales en relación con la investigación pedagógica y disciplinar, junto con sus expectativas profesionales. Así, los estudiantes hacen un primer acercamiento a la problemática en la que les gustaría trabajar en su práctica pedagógica (la cual se lleva a cabo en semestres posteriores).

El APP se formuló en tres etapas: en la primera, seleccionaron la temática, para lo cual realizaron una reflexión sobre su desarrollo durante sus cinco semestres de carrera en cuanto a la investigación pedagógica y disciplinar. Asimismo, plantearon una pregunta problema, producto de sus reflexiones. En la segunda etapa reconstruyeron el problema y avanzaron en el planteamiento del proyecto; para esto, los formadores realizaron un acompañamiento en los ejercicios de metarreflexión, trascendiendo la experiencia, hasta ubicar el tema del APP en los ámbitos académico, institucional e investigativo. En la tercera etapa, ubicaron su planteamiento en un marco de "debates conceptuales” y, finalmente, definieron objetivos, métodos de trabajo y cronograma para el desarrollo del proyecto.

\section{Aportes formativos del diseño de unidades didácticas (quinto semestre del Proyecto Curricular de Licenciatura en Biología)}

Respecto a los componentes del Conocimiento Profesional del Profesor de Biología, la tendencia corresponde a una visión integradora. Los estudiantes en su mayoría consideran que para enseñar biología se requiere integrar los conocimientos pedagógico, biológico, didáctico y humanístico, los cuales se construyen durante la formación como docentes; incluso en el G1 destacan que el profesor ha de contar con un conocimiento ético-político. 
Grupo 1: [Justificación de la UD] El proceso de indagación posibilita tomar conciencia de la importancia y trascendencia de la labor del maestro como sujeto ético y político y la responsabilidad que tiene en la construcción de sujetos y, por tanto, de la sociedad, a partir de la una disciplina como la biología.

Además, coinciden en que la integración de dichos saberes permite desarrollar las habilidades propias del ejercicio docente, como la estructuración de objetivos, actividades, contenidos y evaluación. Particularmente en el G2 se manifiesta que la integración de esos conocimientos le permite al profesor de biología realizar la transposición didáctica.

Esta perspectiva integradora es relevante, pues no sólo se considera que para enseñar biología basta con saber la materia, además es necesario tener Conocimiento Didáctico del Contenido Biológico (CDCB), para cuya construcción se demandan los conocimientos mencionados. Cabe aclarar que en ningún momento los docentes en formación se refieren de manera explícita al concepto CDCB, pero sí implícitamente, es decir, podría formar parte de su epistemología personal, y esto en alguna medida genera una reflexión al respecto y una concienciación en el sentido en que parte de la profesionalidad del profesor consiste en poseer ese conocimiento didáctico.

Llama la atención que los docentes en ejercicio hagan énfasis en la fuente académica sin tener en cuenta los conocimientos experienciales del profesor. $\mathrm{Al}$ respecto, compartiendo los planteamientos de Tardif (2004), se considera esencial que los docentes en formación no desconozcan que la práctica docente constituye una fuente de conocimiento didáctico y para su construcción es menester una reflexión y una sistematización.

En cuanto a las finalidades de la enseñanza de la biología, se plantea que la visión mayoritaria de los futuros licenciados se aproxima a una perspectiva significativa (de acuerdo con la hipótesis de progresión propuesta por Valbuena, 2007) en el sentido que destacan la importancia de:

- Enseñar conceptos biológicos fundamentales de manera integrada que corresponda con la naturaleza sistémica de lo vivo.

- No reducir la enseñanza a los conceptos biológicos y, en contraste, interrelacionar dichos conceptos con procedimientos, actitudes y valores.

- Interrelacionar contenidos procedimentales, conceptuales y actitudinales, con la consecuente concienciación sobre la aplicabilidad de la biología a la vida cotidiana y a la conservación y el provechamiento sostenible de los recursos naturales.

Los estudiantes del G2 plantean que para promover la integración de conceptos, valores, actitudes y procedimientos en el aula se requiere una transposición didáctica. Esto implica que la biología que se enseña, es decir, la "biología escolar", 
ha de ser significativa, y para lograr su construcción el profesor demanda (como lo desarrolla García, 1998) la identificación, la transformación y la integración de saberes, creencias y conocimientos de diferentes orígenes (académico, cultural, experiencial, cotidiano, institucional). Otro aporte por destacar es considerar como finalidad de la enseñanza de la biología, la inclusión como contenido formativo para los escolares de algunos elementos histórico-epistemológicos.

$\mathrm{Al}$ respecto, el grupo 2 afirma: “[...] se ha pretendido vincular los conocimientos científicos con contenidos procedimentales que contextualicen ese saber y fomenten una construcción de los conceptos mucho más personal por parte del estudiante”.

En lo que atañe a la enseñanza de la biología, se da importancia a los referentes teóricos pedagógicos y biológicos. Según los docentes en formación, la enseñanza está orientada en gran medida por los modelos pedagógicos (cambio conceptual y aprendizaje por investigación para el caso del G1 y constructivismo para el caso del G2); así, justifican la manera en que planifican la UD con los elementos conceptuales de dichos modelos.

Grupo 1: Para establecer un cambio conceptual en los estudiantes, se tendrán en cuenta unas preguntas [de indagación de ideas] que aparecen al inicio de la unidad didáctica y que se retomarán al finalizar la misma, es decir, que serán parte de la evaluación, esto se establece como parámetro para evidenciar el cambio conceptual.

La planificación de la enseñanza también está orientada significativamente por los referentes teóricos de la biología; así, cobra importancia la visión integral de lo vivo en la estructuración de contenidos, actividades y objetivos. En todos los casos, los futuros profesores proponen trabajos prácticos de laboratorio, lo cual se puede relacionar con el tipo de conceptos que se pretenden enseñar, articulados con procesos fisiológicos que resultan abstractos para los alumnos de secundaria.

Con base en lo anterior, se considera importante que los futuros licenciados, desde el principio del proceso formativo observado, trasciendan una enseñanza de la biología -centrada en la transmisión de lo disciplinar-, al considerar no sólo los conceptos estructurantes biológicos desde la perspectiva sistémica, sino muy especialmente los referentes pedagógicos, así como las características de los alumnos y su contexto. Vale la pena subrayar que para los estudiantes del G2, al enseñar biología en la escuela, se integran dos tipos de conocimientos: el científico y el cotidiano. En contraste, los estudiantes del otro grupo no hacen explícitas las características del conocimiento que se construye en el aula cuando se enseña la asignatura. 
El grupo 2 plantea como objetivo específico formativo: "Fomentar un conocimiento escolar a partir de las relaciones que se establecen entre los diferentes tipos de conocimientos que confluyen en el aula de clase".

La visión integradora del G1 sobre el conocimiento escolar (CE) se aproxima al nivel de referencia propuesto por Valbuena (2007) al no reducir el CE a la simplificación o resumen del Conocimiento Biológico; sin embargo, además de la fuente científica, los futuros profesores sólo incluyen la cotidiana (las creencias de los alumnos acerca de los conceptos implicados en la enseñanza de la fotoirritabilidad), desconociendo así la importancia de conocimientos como el contextual y el psicopedagógico.

De acuerdo con los planteamientos de autores como García (1998), Martín del Pozo y Rivero (2001) y Valbuena (2007), el CE posee una epistemología propia que lo diferencia de los conocimientos de los cuales se origina. Así, en la formación docente resulta trascendental hacer explícito que la biología que se enseña en la escuela no es la misma que producen los científicos y se diferencia de las creencias del lego acerca de los fenómenos vivientes, siendo así emergente a partir de la transformación e integración de las anteriores, para lo cual el profesor requiere tener presente e integrar aspectos particulares propios de los contextos, como la cultura, las costumbres, las normas, etc., tanto nacional como local e institucional, igual que los alumnos, sus intereses, características propias de la edad, etc.

Es decir, es relevante que el profesor sea consciente del conocimiento que le permite realizar transformaciones e integraciones requeridas para la construcción del CE, principalmente del CDCB. Esto hace que el docente identifique dificultades, potencialidades, recursos, finalidades, contenidos y estrategias de enseñanza buscando que los alumnos adquieran un conocimiento sobre la biología no lejano de las explicaciones cotidianas, ni de las propias de esta disciplina científica, de manera que les aporte en la solución de problemas de la cotidianidad.

Respecto al aprendizaje de la biología, los dos grupos se aproximan a referentes constructivistas. En los dos casos destacan la importancia de socializar los aprendizajes, hecho coherente con las actividades de enseñanza que proponen, en las cuales se promueve compartir y discutir puntos de vista y resultados de los trabajos y se proyecta indagar las ideas de los alumnos y trabajar con ellas. En ambos grupos, se destaca la importancia de indagar las concepciones de los alumnos acerca de los conceptos biológicos por enseñar. En el G1 se hace énfasis en el cambio conceptual, y esto implica sustituir las ideas erróneas de los alumnos, las cuales constituyen un obstáculo para el proceso de enseñanza-aprendizaje:

Las ideas alternativas de los estudiantes están fuertemente arraigadas y son bastante persistentes como para obstaculizar la comprensión de los nuevos conceptos o en nuestro caso dificultar un cambio conceptual en el sentido estricto de la palabra (Grupo 1). 
En lo referente a la evaluación de los aprendizajes, los dos grupos coinciden en una perspectiva corroborativa del aprendizaje. Así, planean instrumentos para evaluar a los alumnos al iniciar y al finalizar la UD en el sentido de comprobar que hayan modificado las concepciones iniciales sobre la temática biológica. Esta visión dista un poco del nivel de referencia propuesto por Valbuena (2007) en el que se asume la evaluación como elemento regulador del proceso de enseñanza-aprendizaje, que constituye una de sus partes consustanciales.

Una vez revisados los programas curriculares de los diferentes seminarios de pedagogía y didáctica del PCLB, se ha evidenciado que no se aborda como contenido curricular el tema de la evaluación de los aprendizajes. Si bien es un asunto presente permanentemente en las prácticas pedagógicas de los diferentes cursos como objeto de discusión y reflexión, se considera que esto no es suficiente, lo que requiere en efecto ahondar en la fundamentación teórica, máxime cuando ésta representa un constituyente del Conocimiento Didáctico del Contenido (Magnusson, Krajcik y Borko, 1999; Valbuena, 2007).

Una coincidencia de los dos grupos analizados es la perspectiva integradora de los contenidos de enseñanza. Así, los futuros profesores no caen en el reduccionismo conceptual; por el contrario, cuando planifican la enseñanza, además de conceptos, incluyen e interrelacionan actitudes, valores y procedimientos.

El maestro realiza una charla donde aborde de manera más completa en las relaciones entre la fotoirritabilidad y el estudiante, a partir de las enfermedades que trae la exposición prolongada a la radiación solar y la activación génica. De esta manera se relaciona un conocimiento científico con uno cotidiano, que es más cercano a la clase y permite mayores construcciones y actitudes de autoestima, salud y bienestar [...] (Grupo 2).

Dicha integración deriva de la reflexión pedagógica y didáctica que media constantemente la formulación de la UD. Así, justifican la organización de los contenidos de enseñanza teniendo en cuenta los modelos pedagógicos, los intereses y las características cognitivas de los estudiantes, los conceptos estructurantes y el contexto. En efecto, la estructuración de contenidos de enseñanza constituye un componente esencial del CDCB.

En la enseñanza de ciencias como la biología, en las cuales existe una vasta cantidad de conceptos y datos, es preciso que el profesor seleccione y secuencie los conceptos estructurantes de la materia, al igual que procedimientos referentes a las ciencias y a esta asignatura, relacionándolos con aspectos relativos a la vida cotidiana de los alumnos, para así incorporar contenidos actitudinales y valores que aporten significatividad al aprendizaje.

La principal dificultad de enseñanza de la biología -identificada por los docentes en formación- consiste en el abordaje analítico de lo vivo que suele aparecer en los 
libros de texto. Este componente del CDCB es importante a la hora de planificar la enseñanza, dado que permite estructurar de manera más integrada los conceptos correspondientes a los procesos biológicos. Asimismo, reconocemos que un obstáculo para la enseñanza de la biología es considerar al organismo como una máquina que funciona gracias a la acción aditiva de los diferentes órganos y de los diferentes procesos, de ahí la dificultad, por ejemplo, de establecer relaciones entre los sistemas digestivo y circulatorio de un animal (Gagliardi, 1986).

En relación con la perspectiva de estudio de la biología, identificamos en G1 y G2, visiones integradora y holística, respectivamente, al considerar lo vivo como un todo, incluso como un sistema.

El tema de ciclo de vida de la(s) célula(s) de los organismos vivos, relacionada con factores como la nutrición, la temperatura, entre otros y diferentes sistemas de control (genético, enzimáticos, hormonales) principalmente. La integración de éstos permite al organismo, bien sea unicelulares o pluricelular, la regulación y auto-mantenimiento, constituyéndolo como patrón dada su presencia en todos los sistemas vivientes (Grupo 1).

Este referente se mantuvo en todo el proceso formativo y se relaciona con la perspectiva teórica del eje curricular, dinámica y mantenimiento de los sistemas, la cual se concentra en lo sistémico. Dichas perspectivas, holística e integral, consideran que no es suficiente estudiar por separado las partes para dar cuenta de la complejidad de la naturaleza viviente; por tal razón, resulta necesario estudiar la naturaleza viviente desde una perspectiva sistémica, enfocándose en las interacciones a diferentes niveles en los sistemas vivos (Valbuena, 2007; Sánchez, 2007).

En cuanto a la experimentación y observación en la biología, G2 se refirió a este aspecto, pues considera que los fenómenos naturales se pueden explicar a partir de su observación. Al respecto, se considera que para un profesor de biología es importante tener en cuenta que la producción de CB no se puede reducir a lo sensorial, dado que en esta ciencia es menester recurrir a las narrativas históricas, especialmente en el campo de la biología evolutiva (Jiménez, 2003). Tampoco se puede perder de vista que la temática de la UD (fotoirritabilidad) corresponde más a lo que Caponi (2003) referencia como biología funcional, en la cual la experimentación cobra relevancia.

Cabe señalar que los futuros profesores del G1 hacen énfasis en la experimentación al proponer actividades de enseñanza como el diseño de experimentos con animales, entre otras. Es importante resaltar que para un profesor de biología cobra importancia tener claridad sobre las especificidades en la manera en que se produce el CB, el cual está relacionado con lo que autores como Grossman (1990) denominan la estructura sintáctica del conocimiento disciplinar. En la misma dirección, Bromme (1988), Porlán, et al. (1996), García (1998), Porlán y Rivero (1998), y Valbuena 
(2007), entre otros, identifican el conocimiento epistemológico de la disciplina como conocimiento metadisciplinar que incide significativamente en la manera en que el profesor orienta sus prácticas, cómo asume el conocimiento, cómo estructura las actividades y los contenidos de enseñanza.

En ese sentido, se requiere incluir como contenido pedagógico, en la formación inicial de profesores de biología, la discusión epistemológica sobre las particularidades de la asignatura en cuanto a la producción de su conocimiento. Así, por ejemplo, en la formación de profesores es preciso retomar autores como Canguilhem (1976), Mayr (1998), Jiménez (2003) y Caponi (2003), a fin de precisar las limitaciones que tiene el experimento en biología, dadas las particularidades de lo vivo respecto a características, como la variabilidad, la irrepetibilidad y la totalidad, las cuales generan la imposibilidad de controlar variables, de predecir con exactitud, y de manejar réplicas en el trabajo en laboratorio. Este tipo de debates puede contribuir a que el docente en formación perciba que la enseñanza de la biología demanda particularidades.

En cuanto a la estructura conceptual biológica, en el ejercicio formativo de diseño de una UD, resulta difícil detectar los referentes generales de la organización de los conceptos de la biología, aunque sí es posible identificar la manera en que los estudiantes relacionan los conceptos implicados en los temas que pretenden enseñar. Los estudiantes de G1 y G2 identifican conceptos integradores, incluso los del G1 reconocen conceptos estructurantes. Esto resulta importante, dado que un problema en la enseñanza de la biología radica en los esfuerzos del docente centrados en cubrir gran cantidad de contenidos para "cumplir a cabalidad el programa" (Valbuena, 2007), en ocasiones, sin cuestionar el porqué de los contenidos que se enseñan.

Como se puede apreciar, la actividad formativa del diseño de la UD posibilitó a los futuros profesores identificar los componentes del CPPB, así como reflexionar y poner en práctica los factores que constituyen el Conocimiento Didáctico del Contenido Biológico. Al respecto, vale la pena resaltar que el equipo de formadores de quinto semestre realiza un seguimiento continuo mediante el desarrollo de tutorías a los grupos de trabajo, tanto en las asignaturas específicas como en espacios destinados concretamente para la tutoría. En dichas asesorías, los formadores retroalimentan los distintos aspectos de las propuestas (título, propósitos, antecedentes, estructuración de redes de conceptos y de contenidos de enseñanza, actividades de evaluación y de enseñanza, etc.).

Tal como se había planteado anteriormente, durante el proceso de diseño de la UD, los futuros docentes elaboran un documento en el que lo central es la reflexión a partir del aporte de la actividad en la formación como profesores de biología.

Dicho ejercicio representa una dificultad en el estudiante, especialmente al inicio del proceso; sin embargo, logran destacados avances como a) explicitar el porqué de la importancia de esa actividad en su formación; b) argumentar la organización de conceptos biológicos implicados mediante la elaboración de una red conceptual, la cual representa un insumo importante en la organización de contenidos de ense- 
ñanza; c) sustentar la estructuración de la red de contenidos de enseñanza, lo que llega a trascender los conceptos biológicos al incorporar procedimientos y actitudes, para lo cual no se limitan a la lógica disciplinar biológica, sino que tienen en cuenta aspectos como el contexto, las características de los alumnos y los propósitos de enseñanza, entre otros aspectos.

En efecto, el diseño de la UD posibilitó a los docentes en formación argumentar que el conocimiento que se enseña en una clase de biología no es el mismo que producen los científicos, sino que posee un estatus propio y corresponde al conocimiento biológico escolar ("biología escolar"); además, fue posible que los futuros profesores explicitaran que para enseñar la asignatura, es decir, para producir didácticamente tal materia, es imperiosa una transformación didáctica.

\section{Aportes formativos de la formulación de anteproyectos de práctica pedagógica (sexto semestre del proyecto curricular licenciatura en biología)}

Durante el proceso de la formulación del APP, los futuros profesores -como producto de su reflexión- manifiestan en sus informes que para enseñar biología, además de tener idoneidad en la disciplina biológica, es preciso conocer las características de los estudiantes y los elementos contextuales y experienciales. Es decir, respecto a los componentes del Conocimiento Profesional del Profesor de Biología, no existe un reduccionismo biológico, pues se hace énfasis en otros conocimientos y saberes que confluyen de manera necesaria en la enseñanza. Esto explicita un estudiante haciendo referencia a sus prácticas educativas:

De la experiencia vivida dentro de las tan llamadas "Chiquitecas" me queda solo resaltar la importancia que tiene el contexto socio-cultural que envuelve e influencia la vida escolar, una realidad que como maestros es necesario, conocer, reflexionar y problematizar (Estudiante 4).

Asimismo, se manifiesta que en los aspectos relacionados con los procesos de enseñanza-aprendizaje, como definición de objetivos, contenidos y estrategias de enseñanza, es necesario tener en cuenta la realidad en que se encuentran involucrados los estudiantes, así como sus intereses.

La mayoría de los profesores de ciencias, investigadores en educación, y la comunidad educativa en general, se preocupan por indagar y darle solución a las problemáticas que se suceden al interior del aula de clases; problemas tales como, los objetivos a trabajar en una disciplina como la Biología, la toma de decisiones acerca de los contenidos que guiarán la clase; y más importante aún, los intereses, ya que esto trae consigo, el diseño e implementación de es- 
trategias educativas, gracias a que tanto el sujeto como su forma de aprender son diversas. Es por tanto, que hablar de estrategias educativas que se adapten a las múltiples necesidades de nuestros estudiantes, se convierte entonces, en una cuestión a la que muchas investigaciones tratan de darle alternativas que la viabilicen (Estudiante 1).

Lo anterior señala que, desde una perspectiva deseable, no basta con que el docente maneje los conocimientos disciplinares de la materia que enseña, pues además de éstos debe contar con elementos pedagógicos, didácticos, curriculares y contextuales, enmarcados como componentes de su CPP (Valbuena, 2007). También cabe resaltar que aunque estos componentes inicialmente provienen de la fuente académica, es decir, de la formación inicial que han tenido los futuros docentes, es fundamental reconocer la importancia que tiene la fuente experiencial, cuando se ponen en juego en determinado contexto bajo condiciones particulares. Para el caso expuesto por el estudiante, los referentes se encuentran en los tres informes entregados, hecho que demuestra una constante.

Con relación a las finalidades de la enseñanza de la biología, mencionadas por tres estudiantes, éstas corresponden con una perspectiva significativa-contextual, puesto que se establecen como propósitos: incluir en la enseñanza contenidos de tipo conceptual, procedimental y actitudinal, haciendo especial énfasis en este último, pues continuamente se resalta lo axiológico como uno de los logros que se deben promover en el aprendizaje de la biología. Por ejemplo, uno de los estudiantes explicita que dichas finalidades deben estar orientadas a generar actitudes y valores sobre el reconocimiento de la importancia de la diversidad y, por tanto, sobre la conservación de especies endémicas, en especial ranas, con el fin de fomentar la valoración y el respeto por la naturaleza. En cuanto a la justificación del anteproyecto, el Estudiante 5 afirma:

El objetivo es el siguiente: fortalecer la identidad nacional a través de la formulación y el desarrollo de acciones, actitudes y valores que permitan el reconocimiento y promoción de nuestra diversidad natural y de su carácter cultural. Aunque yo le agregaría la conservación como parte importante del reconocimiento y promoción de nuestra diversidad natural y de su carácter cultural.

Otra coincidencia en los estudiantes radica en articular lo que se enseña con su aplicabilidad en la sociedad. Así, destacan la importancia de favorecer las relaciones entre hombre, naturaleza y sociedad, buscando para el caso del contexto colombiano una identidad nacional, con énfasis en la diversidad biológica y cultural; además, resaltan la incidencia de los aprendizajes en el desarrollo del país desde lo social, lo ambiental y lo educativo. Otra de las finalidades mencionadas es la iden- 
tificación de problemas a nivel biológico y social, y la vinculación de la comunidad para su solución, en la que el contexto es fundamental; así, destacan la labor del profesor más allá del aula de clase posibilitando su acción social, la cual se desarrolla gracias al reconocimiento que éste haga de las necesidades sociales y culturales de la comunidad con la que trabaje. El Estudiante 2 afirma lo siguiente refiriéndose a la propuesta:

De esta forma se intenta proyectar un programa que contribuya a la construcción de valores y actitudes en una comunidad específica que se encuentre afectada por problemas ambientales de carácter natural pero también social, donde pueda generarse una perspectiva sistémica del ambiente para comenzar a esbozar posibles soluciones por parte de los mismos habitantes.

En términos generales, lo anterior refleja la importancia que se ha otorgado en el proyecto curricular, a la necesidad que tienen los profesores de conocer más que otros sobre su materia, en particular cuestiones referidas a normas sociales, así como la utilidad y la relevancia para la vida cotidiana, en contraste con quienes defienden que un docente requiere conocer menos del contenido que otros especialistas en la misma materia, y que, supuestamente, sólo basta con lo que señalan el currículo y los libros de texto, según Marcelo (2005).

Así, en las diferentes propuestas se puede identificar la superación de la visión de la enseñanza de la biología como transmisión de saberes, la cual consiste en explicar los contenidos biológicos, fundamentalmente los conceptuales, como están establecidos en los libros de texto y en los programas curriculares. Desde esta perspectiva, los protagonistas de la enseñanza son el profesor y los contenidos, lo que conduce a que las decisiones en el aula sean tomadas por el docente (Valbuena, 2007).

Los futuros profesores mencionan algunos elementos que, a juicio propio, contribuyen a abandonar los tradicionales modelos de enseñanza, los cuales, según Tardif (2004), pretenden que el profesor se limite a implementar la producción de los especialistas — quienes determinan qué tipo de contenidos disciplinares debe aprender el profesor, hecho que produce una relación vertical entre los gestores del conocimiento y los ejecutores del mismo- o, por otro lado, consideran que para enseñar basta con contar con las habilidades personales y apoyarse en la experiencia sin fundamentarse en un saber riguroso.

Otro aspecto para destacar en la formulación de los APP consiste en que los futuros docentes no asumen la enseñanza de una manera espontánea ni activista, en el sentido de limitarse a proponer una gran cantidad de actividades; por el contrario se apoyan en referentes teóricos de la pedagogía. En una de las propuestas, la cual se basa en una perspectiva constructivista y un modelo de aprendizaje significativo, coherente con el propósito, se destaca la importancia de incorporar en el proceso de enseñanza-aprendizaje el trabajo con ideas previas, imaginarios y desequilibrios 
cognitivos. En otro caso, el docente en formación formula su propuesta en función de la realidad de los estudiantes en cuanto a sus intereses y, muy especialmente, a sus limitaciones físicas (visuales para este caso); su propuesta se centra en la implementación de estrategias metodológicas que no excluyan a estudiantes con limitación visual, por ejemplo, de una didáctica multisensorial.

Es así que para retomar la problemática de la observación en los estudiantes con necesidades especiales en cuanto a $\mathrm{LV}$ se refiere y estudiantes sin problemas de visión, se debe reconocer no solo el problema, sino también una estrategia que permita manejar de mejor manera la anterior situación; por ende considero que retomando las palabras del Doctor Soler: "La observación tiene que ser multisensorial" (Estudiante 4).

En cuanto a los contenidos de enseñanza, los APP sistematizados abordan la construcción de conceptos, el desarrollo de actitudes de respeto y valoración hacia los seres vivos y su entorno, y la promoción de habilidades propias del trabajo científico. En cuanto a la justificación del proyecto, el Estudiante 5 afirma:

\begin{abstract}
Además otro de los motivos de realizar este trabajo es el desconocimiento que se tiene acerca de la existencia de ranas en la Sabana de Bogotá, lo cual me lleva a pensar que por eso no se les "cuida" y sería interesante que la conservación de este anfibio se convirtiera en algo cotidiano para el estudiante ya que no sólo se preocupará por el cuidado de las ranas sino también de otras especies endémicas de la Sabana de Bogotá.
\end{abstract}

Resulta importante identificar los diversos tipos de contenidos de enseñanza de las ciencias, con el fin de promover la idea que la enseñanza no se restringe a la enseñanza exclusiva de conceptos, teorías o leyes de una disciplina, sino que, además de éstos, existe una serie de contenidos procedimentales, bien sea de orden algorítmico o cognitivo y unos contenidos actitudinales, relacionados con conductas y valoraciones que realizan los sujetos (Coll et al, 1992).

En cuanto al Conocimiento Biológico, las propuestas presentan una perspectiva interdisciplinar; así, en el concepto de ambiente confluyen la biología, las humanidades y las ciencias sociales. Sin embargo, los informes no aportan suficientes elementos para caracterizar esta categoría de investigación. De hecho, llama la atención que los docentes en formación presenten pocos elementos biológicos en sus APP. Pareciera que en una de ellas predomina una visión sobre la producción del $\mathrm{CB}$, centrada en un único método científico, en la que observación tiene un lugar privilegiado mediante ésta se puede dar cuenta del fenómeno en estudio, aclarando que la observación, además de lo visual, implica los otros sentidos. 
Es importante añadir que gracias a mi discapacidad la pregunta problema posiblemente va dirigida a estudiantes que como yo, les es complejo entender una ciencia que está netamente de lo visual como la biología; sin embargo, considero que la observación no puede prescindir esta ciencia, comprende todos los demás sentidos de los que estamos provistos y así mismo se puede potencializar la capacidad de observación desde otras perspectivas (Estudiante 4).

Es posible que las escasas referencias al Conocimiento Biológico se deban a las intencionalidades mismas de los formadores, puesto que al solicitar la reflexión y la propuesta pedagógica centran los contenidos del informe en lo pedagógicodidáctico y en lo curricular, haciendo de lado lo biológico, lo cual debe ser revisado puesto que un contenido formativo esencial es el disciplinar. Puesto que nuestra labor es formar a los formadores para que construyan un Conocimiento Didáctico del Contenido para enseñar, resulta importante solicitar a los futuros docentes, en este tipo de actividades, hacer explícitas las relaciones de integración del conocimiento pedagógico y didáctico de la materia por enseñar con el conocimiento del contenido, el cual debe incluir los componentes sustantivos y sintácticos (Grossman, 1990).

De esta manera, los profesores en formación podrían consolidar un conocimiento experto del contenido que pretenden enseñar, para que cuando se encuentren en ejercicio puedan llevar a cabo una enseñanza que favorezca una mejor comprensión por parte de los alumnos, como lo plantea Marcelo (2005): Existen múltiples ejemplos de investigaciones que muestran que poseer un dominio profundo de una disciplina lleva a una actividad docente más centrada en problemas, con mayor participación de los alumnos, menores digresiones y preguntas de alto orden cognitivo.

\section{Conclusiones}

La actividad formativa diseño de unidades didácticas para la enseñanza de temáticas biológicas:

1. Favorece en los futuros docentes el reconocimiento de los componentes del CPPB y la importancia de integrarlos.

2. Permite a estudiantes-profesores reconocer que el conocimiento que se construye en el aula de clase, cuando se enseña y se aprende biología, difiere del conocimiento producido por la comunidad de biólogos, pues se trata de un conocimiento escolar epistemológicamente diferenciado, que ha de producirse didácticamente a partir de las transformaciones e integraciones de diferentes saberes y conocimientos que confluyen en la escuela (es decir, a partir de la transformación didáctica). 
3. Posibilita la identificación de conceptos estructurantes (mediante realización y sustentación de redes conceptuales) y su transformación e integración con otros saberes y conocimientos para la organización de los contenidos de enseñanza -que trascienden lo meramente conceptual- estructurados, en principio, en redes y materializados, a la postre, en la planificación de contenidos y actividades de enseñanza.

La actividad formativa formulación de Anteproyectos de la Práctica Pedagógica:

1. Posibilita en futuros profesores la reflexión sobre sus experiencias, tanto en el ámbito académico (con especial énfasis en su transcurso durante los cinco anteriores semestres en el Proyecto Curricular de Licenciatura en Biología) como personal, y la identificación de su incidencia en la formación docente y en la proyección profesional a corto y mediano plazo (práctica docente y ejercicio como profesores de biología).

2. Permite a docentes en formación contextualizar sus propuestas pedagógicas e identificar su relevancia en un proceso significativo de enseñanza-aprendizaje.

3. Se centra en reflexiones sobre la formación docente, sin profundizar en los aspectos biológicos.

Las actividades formativas diseño de unidades didácticas para la enseñanza de temáticas biológicas y formulación de Anteproyectos de la Práctica Pedagógica del Proyecto Curricular de Licenciatura en Biología:

1. Dada su estructuración y dinámica de formadores implicados en los procesos, promueven el desarrollo de habilidades de reflexión y metarreflexión, en los docentes en formación, centradas en concebirse como futuros profesores por e identificar y regular los procesos de formación docente. Estas habilidades son fundamentales para la construcción del CPPB dado que permiten integrar reguladamente los diferentes componentes de dicho conocimiento.

2. Aportan elementos a futuros docentes para identificar e integrar componentes diferentes al Conocimiento Biológico en la enseñanza de la asignatura, como los contextuales, los saberes populares, los referentes curriculares y las características de los alumnos.

3. Hacen que los docentes en formación involucren de manera integrada, en los procesos de enseñanza, los contenidos conceptuales, procedimentales y actitudinales, lo cual contribuye a un aprendizaje más significativo en los alumnos. 
4. Promueven en futuros profesores la necesidad de fundamentarse teóricamente, en particular, en lo concerniente a los referentes pedagógicos (especialmente los modelos pedagógicos) y biológicos, contribuyendo así al "empoderamiento" de la profesión docente al evidenciar que ésta demanda una sólida formación.

\section{Referencias bibliográficas}

Astolfi, J. (1997). Conceptos clave en la didáctica de las disciplinas. Sevilla: Díada.

Bardín, L. (1977). Analyse de contenu. París: Presses Universitaries de France.

Canguilhem, G. (1976). El conocimiento de la vida. Barcelona: Anagrama.

Caponi, G. (2003). Experimentos en biología evolutiva: ¿qué tienen ellos que los otros no tengan? Episteme, Porto Alegre, 16, 61-97.

Carlsen, W. (1999). Domains of teacher knowledge. En J. Gess-Newsome y N. Lederman (Eds.) (s. f.), Examining pedagogical content knowledge. The construct and its implications for science education (pp. 133-144). Londres: Kluwer Academic Publishers.

Coll, C., Pozo, I., Sarabia, B. y Valls, E. (1992). Los contenidos en la reforma. Enseñanza y aprendizaje de conceptos, procedimientos y actitudes. Madrid: Santillana.

Departamento de Biología. (1999). Proyecto Curricular de Licenciatura en Biología. Bogotá: Universidad Pedagógica Nacional. Documento inédito.

García, E. (1998). Hacia una teoría alternativa sobre los contenidos escolares. Sevilla: Díada.
GAGLIARDI, R. (1986). Los conceptos estructurantes en el aprendizaje por investigación. Enseñanza de las Ciencias, 4(1), 30-35.

Gess-Newsome, S. \& Lederman, N. (1993). Preservice biology teachers' knowledge structures as a function of professional teacher education: a yearlong assessment. Science Education, 77(1), 25-45.

Gess-Newsome, S. \& Lederman, N. (1995). Biology teachers' perceptions of subject matter structure and its relationship to classroom practice. Journal of Research in Science Teaching, 32(3), 301-325.

Gess-Newsome, S. \& Lederman, N. (ed.). (1999). Examining pedagogical content knowledge. The construct and its implications for science education. Londres: Kluwer Academic Publishers.

Gil, D., Carrascosa, J. y Martínez, F. (1999). El surgimiento de la didáctica de las ciencias como campo específico de conocimientos. Revista Educación y Pedagogía, 11(25), 13-66.

Gil-Pérez, D., Carrascosa, J. y MarTíNEZ, F. (2000). La didáctica de las ciencias. Una disciplina emergente y un campo específico de investigación. En F. Perales, y P. Cañal (s. f.). Didáctica de las ciencias experimentales. Teoría y práctica de la enseñanza de las ciencias (pp. 11-34). Alcoy: Marfil. 
Grossman, P. (1990). The making of a teacher. Teacher knowledge and teacher education. Nueva York: Teachers College, Columbia University.

Gutiérrez, A. (2008). Uso de estrategias metacognitivas y conocimiento profesional del profesor. Tesis de maestría. Bogotá: Pontificia Universidad Javeriana.

HashweH, M. (1987). Effects of subject-matter knowledge in the teaching of biology and physics. Teaching \& Teacher Education, 3(2), 109-120.

Hauslein, P., Good, R. \& Cummins, C. (1992). Biology content cognitive structure: from science student to science teacher. Journal of Research in Science Teaching, 29 (9), 939-964.

Hoz, R., Tomer, Y. \& Tamir, P. (1990). The relations between disciplinary and pedagogical knowledge and the length of teaching experience of biology and geography teachers. Journal of Research in science teaching, 27 (10), 973-985.

Jiménez, M. (coord.). (2003). Enseñar ciencias. Barcelona: Graó.

Lederman, N., Gess-Newsome, S. \& LATZ, M. (1994). The nature and development of preservice science teachers' conceptions of subject matter and pedagogy. Journal of Research in Science Teaching, 31(2), 129-146.

Magnusson, S., Krajcik, J. \& BorKO, H. (1999). Nature, sources, and development of pedagogical content knowledge for science teaching. En J. Gess-Newsome y N. Lederman (Ed.) (s. f.), Examining pedagogical content knowledge. The construct and its implications for science education (pp. 95-132). Londres: Kluwer Academic Publishers.
Marcelo, C. (1999). Cómo conocen los profesores la materia que enseñan. Algunas contribuciones de la investigación sobre conocimiento didáctico del contenido. En L. Montero y E. Vez (Ed.) (s. f.), Las didácticas específicas en la formación del profesorado (pp. 151185). Santiago de Compostela: Tórculo.

Marcelo, C. (2005). La investigación sobre el conocimiento de los profesores y el proceso de aprender a enseñar: Una revisión personal. En A. Perafán y A. Adúriz-Bravo (Comp.) (s. f.), Pensamiento y conocimiento de los profesores. Debate y perspectivas internacionales (pp. 45-61). Bogotá: Nomos.

Martín del Pozo, R. \& Rivero, A. (2001). Construyendo un conocimiento profesionalizado para enseñar ciencias en la educación secundaria: los ámbitos de investigación profesional en la formación inicial del profesorado. Revista Interuniversitaria de Formación del Profesorado, 40, 63-79.

Mayr, E. (1998). Asi es la biología. Barcelona: Debate.

Morine-Dershimer, G. y Kent, T. (1999). The complex nature and sources of teachers' pedagogical content knowledge. En J. Gess-Newsome y N. Lederman (Ed.) (s. f.), Examining pedagogical content knowledge. The construct and its implications for science education (pp. 21-50). Londres: Kluwer Academic Publishers.

Porlán, R. (1998). Pasado, presente y futuro de la didáctica de las ciencias. Enseñanza de las Ciencias, 16(1), 175 185.

Porlán, R., Azcárate, P., Martín del Pozo, R., Martín, J. y Rivero, A. (1996). Conocimiento profesional 
deseable y profesores innovadores: fundamentos y principios formativos. Investigación en la Escuela, 29, 23-38.

Porlán, R., Martín del Pozo, R., Martín, J. y Rivero, A. (2001). La relación teoría-práctica en la formación del profesorado. Sevilla: Díada.

Porlán, R. y Rivero, A. (1998). El conocimiento de los profesores: una propuesta formativa en el área de ciencias. Sevilla: Díada.

Porlán, R., Rivero, A. y Martín Del Pozo, R. (2000). El conocimiento profesional del profesorado sobre la ciencia, su enseñanza y aprendizaje. En: F. Perales y P. Cañal (s. f.), Didáctica de las ciencias experimentales. Teoría y práctica de la enseñanza de las ciencias (pp. 507-533). Alcoy: Marfil.

Putnam, R. \& Borko, H. (1998). Teachers learning: implications of new views of cognition. En B. Biddle et al. (Ed.) (s. f.), International handbook of teachers and teaching: the new synthesis (pp. 35-49). Nueva York: Jossey-Bass.

SÁnchez, P. (2007). Formulación de proposiciones para el estudio de las concepciones sobre el conocimiento biológico en el marco del conocimiento profesional del profesor de biología. Trabajo de grado. Bogotá: Universidad Pedagógica Nacional.

Shulman, L. (1986a). Those who understand: knowledge growth in teaching. Educational Researcher, 15(2), 4-14.

Shulman, L. (1986b). Paradigms and research programs in the study of teaching: a contemporary perspective. En
M. Wittrock (Ed.) (s. f.), Handbook of research on teaching (pp. 3-36). Nueva York: Macmillan.

Schön, D. (1992). La formación de profesionales reflexivos. Hacia un nuevo diseño de la enseñanza y el aprendizaje en las profesiones. Barcelona: Paidós.

TARDIF, M. (2004). Los saberes del docente y su desarrollo profesional. Madrid: Narcea.

Valbuena, E. (2007). El conocimiento didáctico del contenido biológico. Estudio de las concepciones disciplinares y didácticas de futuros docentes de la Universidad Pedagógica Nacional (Colombia). Tesis doctoral. Madrid: Universidad Complutense de Madrid.

Valbuena, E., Castro, A. y Sierra, C. (2006). El conocimiento biológico desde la perspectiva del conocimiento profesional del profesor de biología. Memorias Segundo Congreso Internacional de Enseñanza de la Biología, Neuquén, Argentina.

Valbuena, E., Sierra, C., Castro, A., Rodríguez, O., Castañeda, D., HerNÁNDEZ, D., SÁNCHEZ, P. y GuTiéRREZ, P. (2006). La hipótesis de progresión como perspectiva metodológica para el estudio de las concepciones acerca del conocimiento biológico. Ponencia aprobada para el XL Congreso de la Asociación Colombiana de Ciencias Biológicas.

Veal, W. \& Kubasko, D. (2003). Biology and geology teachers' domainspecific pedagogical content knowledge of evolution. Journal of Curriculum and Supervision, 18(4), 334-352. 



\section{Centro de Documentación en Educación del Icfes}

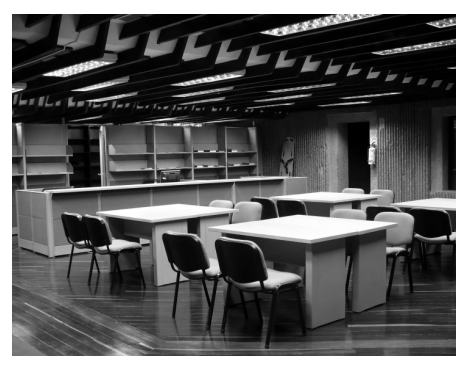

Se inauguró en el Instituto Colombiano para el Fomento de la Educación Superior (ICFES) el nuevo Centro de Información Documental (CID).

En él se podrán encontrar más de 2700 títulos en temas institucionales, resultados y análisis de estudios realizados en relación con las pruebas que aplica el Instituto, como el Examen de Estado, Ecaes, Saber, y pruebas internacionales como PISA, entre otras, así como normatividad vigente $y$, en general, material de interés en el tema educativo.

Con instalaciones modernas, acogedoras y especialmente diseñadas para sus usuarios, el CID cuenta con sala de lectura y un catálogo vía web que pueden consultar desde la página oficial del Instituto (www.icfes.gov.co) estudiantes, docentes, investigadores y toda la comunidad en general interesada en profundizar en los temas referentes a la evaluación educativa.

Uno de los objetivos del CID es apoyar la divulgación y comprensión de dichos temas en el país, por lo cual se brinda a los usuarios la posibilidad de reproducir los documentos que soliciten, así como el préstamo interbibliotecario.

Para mayor información, favor comunicarse al teléfono directo (057-1) 3410341 o al PBX (057-1) 33873 38/60, ext. 1061.

Información remitida por el Icfes 


\section{Normas para la presentación de trabajos}

1. La Revista Colombiana de Educación es una publicación del Centro de Investigaciones de la Universidad Pedagógica Nacional de Colombia, CIUP, orientada principalmente a la divulgación de trabajos de investigación educativa y a estudios teóricos sobre el campo de la educación. A tal efecto, trabaja semestralmente con números temáticos. Se sugiere consultar la temática antes de remitir cualquier contribución.

2. Sólo se recibirán para su publicación trabajos inéditos o con escasa difusión en Colombia, actualizados, y que signifiquen un aporte empírico o teórico de relevancia.

\section{Envío de originales}

3. La recepción de un trabajo no implicará ningún compromiso de la Revista para su publicación. El material enviado no será devuelto.

4. Los trabajos no podrán sobrepasar las treinta páginas y se recibirán en los correos electrónicos de la Revista: rce@pedagogica.edu.co; rceupn@gmail.com, con el siguiente formato: programa Word para Windows, tamaño carta, a espacio y medio, letra arial, tamaño 12. Se establecerán como márgenes del trabajo $3 \mathrm{~cm}$. por cada lado, $2 \mathrm{~cm}$. para encabezado y 2,5 cm. para pié de página. En el cuerpo del texto no deben emplearse ni subrayados ni negritas; en caso de que sea necesario enfatizar una palabra, pueden utilizarse las itálicas o bastardillas.

5. Para las citas bibliográficas y las referencias, se seguirá el estilo de la American Psychological Association, APA. Sus directrices se pueden consultar en la página de la Revista en la dirección http://www. pedagogica.edu.co:8080/portal/contenido.php? esquema=759\# del portal Web de la Universidad Pedagógica Nacional de Colombia.

6. Las notas a pie de página tendrán una secuencia numérica y se debe procurar que sean pocas y escuetas; es decir, que se empleen para hacer aclaraciones al texto y no para citar libros. Los libros consultados sólo aparecerán relacionados en la bibliografía.

7. Todas las páginas deberán estar numeradas, incluyendo las de referencias bibliográficas.

8. En hoja aparte deberán incluirse el nombre del autor, título académico que posee, su dirección postal, institución donde trabaja y cargo que desempeña, así como el número de teléfono, fax y correo electrónico, adicionando las referencias de los artículos publicados en otras revistas científicas, en el transcurso de los dos últimos años.

9. Cada trabajo deberá incluir un resumen del mismo, no mayor de 20 líneas en español y su correspondiente versión en inglés (inclusive el título); al igual que cinco palabras clave (mínimo) en los dos idiomas.

10. El artículo, el resumen y la información del autor, deberán ser acompañados de una carta dirigida al Comité Editorial de la Revista en la cual conste que el texto presentado es original e inédito; es decir, que no ha sido publicado en forma parcial o total en ningún medio impreso o electrónico y que no ha sido sometido a consideración de otra publicación.

\section{Evaluación}

1. El comité de redacción procederá a la selección de los trabajos de acuerdo con los criterios formales y de contenido de esta publicación.

2. El material seleccionado se remitirá a evaluación de pares académicos y posteriormente se someterá a arbitraje para determinar si será publicado y la fecha de publicación.

3. Una vez definida la publicación o no de un texto, se le informará al autor. Los autores de los textos aprobados, deberán autorizar por escrito a la Universidad Pedagógica Nacional para su publicación.

\section{Revista Colombiana de Educación}

División Gestión de Proyectos, Centro de Investigaciones Universidad Pedagógica Nacional,

CIUP.

Calle 127 N.11-20; A. A. 59530; rce@pedagogica.edu.co; rceupn@gmail.com

Tel.: (057-1) 6156531 - 6156512

Suscripción nacional e internacional 